\title{
A case of major lung herniation with minor trauma
}

\author{
Carvalho $\mathrm{AM}^{1 *}$ and Anderson $\mathrm{DA}^{2}$ \\ ${ }^{1}$ Queen's University Belfast School of Medicine, UK \\ ${ }^{2}$ Radiology Department, VA Medical Center, Boise, Idaho, USA
}

\begin{abstract}
We present a case of significant lung herniation which was related to a relatively low acuity injury sustained while moving a small load of bricks. The patient was symptomatic with pain and dyspnea, and chest images revealed a large herniation of the right lower lobe through a defect in the intercostal muscles. Lung herniation is rare but is usually associated with significant trauma or surgery. This degree of injury occurred with minor provocation but caused significant morbidity.
\end{abstract}

\section{Case report}

A 62-year-old man presented to the emergency room with swelling and pain at his right lower costal margin. A sharp tearing pain was reported at the initial injury which occurred while moving bricks 2 days prior. Several hours later, the patient, who is a smoker, had a coughing episode with aggravation of the pain and subsequent development of a soft spongy bulge at the site. The bulge increased in size with subsequent coughing and became severely painful, preventing normal tidal breaths. The patient reduced the swelling by external compression with a broad belt which he wore in order continue working. Although compression was helpful, he was unable to continue work due to intractable pain.

On physical exam, the patient was alert and in no immediate distress. He had a 30 pack-year history of cigarette smoking but was otherwise in good health and used no medications. Vital signs were normal, except for a respiratory rate at 22 /minute with shallow breaths. The patient was holding his right side and had a leather belt tightly bound over his upper abdomen. He was unable to take deep breaths, but there were decreased breath sounds over the right lateral and posterior lung fields. Once the belt was removed, there was no apparent swelling, but a depression was palpated at the lateral aspect between the eighth ninth rib interspace. The patient reported that he could replicate the bulge beneath the skin if he coughed.

A chest $\mathrm{x}$-ray showed an indistinct right basilar density with a potential small effusion possibly related to atelectasis or pneumonia (Figures $1 \mathrm{~A}$ and $1 \mathrm{~B}$ ). There were no evident rib fractures. While preparing for a chest CT, the patient coughed, and the bulge recurred. CT images showed a large herniation of the right lower lobe between the 9 th and 10th rib interspace. The adjacent ribs were separated with interruption of the associated intercostal muscles (Figures $2 \mathrm{~A}$ and $2 \mathrm{~B}$, Figure 3 and Figure 4).

The patient subsequently underwent a right thoracotomy with placement of a Gore-Tex patch to close the defect. A sterile seroma cavity had formed in the chest wall and was excised. The patient has recovered well and has not experienced further pain or recurrence of herniation on follow-up CT (Figure 5).

Pulmonary herniation is a rare condition characterized by protrusion of the lung beyond the thoracic cavity. Less than 300 cases have been reported. Lung herniation is generally caused by increased

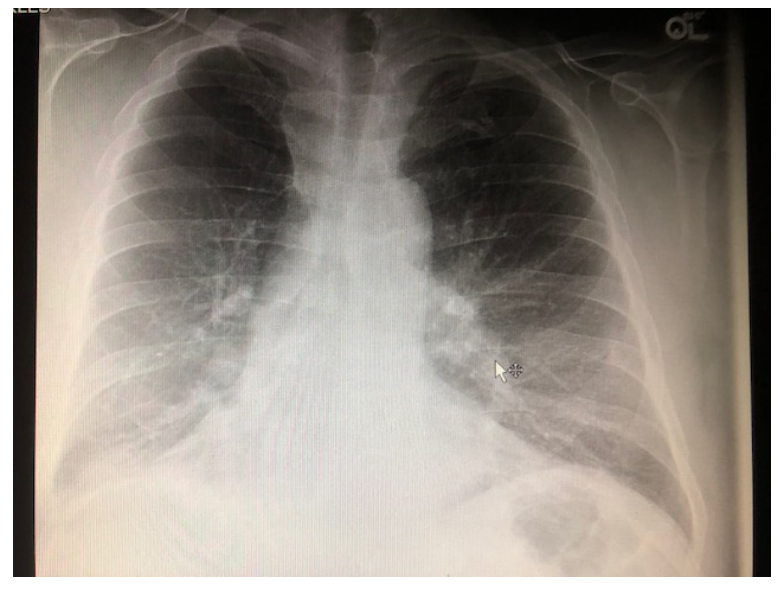

Figure 1A. Admission chest X-ray, PA view showing a vague increased right basilar density thought to be a potential small effusion perhaps related to atelectasis or pneumonia

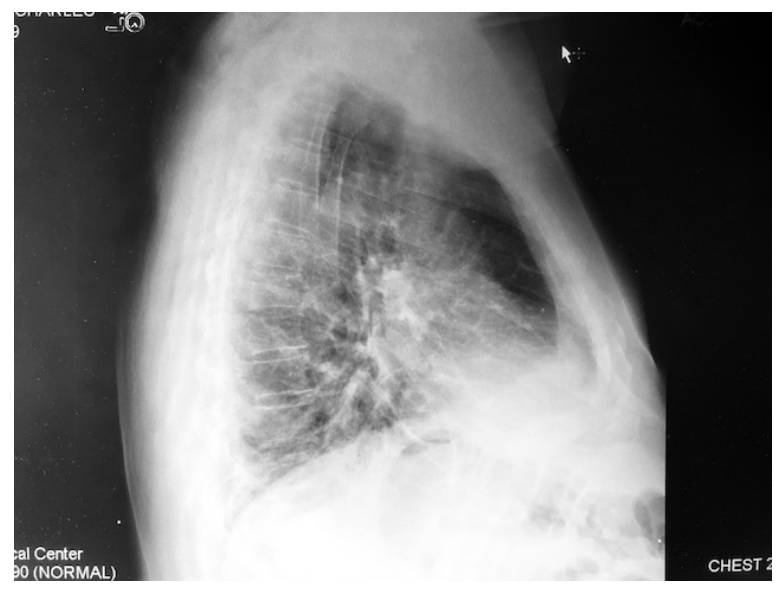

Figure 1B. Admission chest X-ray, lateral view

${ }^{\star}$ Correspondence to: Carvalho AM, Queen's University Belfast School of Medicine, UK, E-mail: Paula.Carvalho@med.va.gov

Received: June 15, 2018 Accepted: June 25, 2018; Published: June 28, 2018 


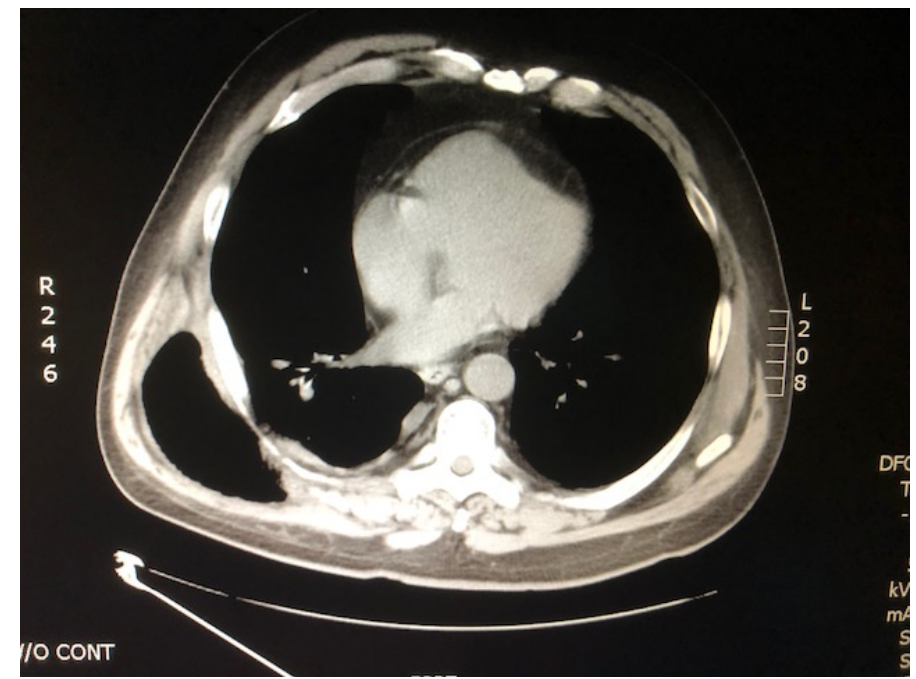

Figure 2A. CT of chest, non-contrast, mediastinal windows. Axial view shows a large herniation of the right lower lobe between the eighth and ninth rib interspace and a chest wall defect. The intercostal muscles in this posterior lateral location are discontinuous over a broad area

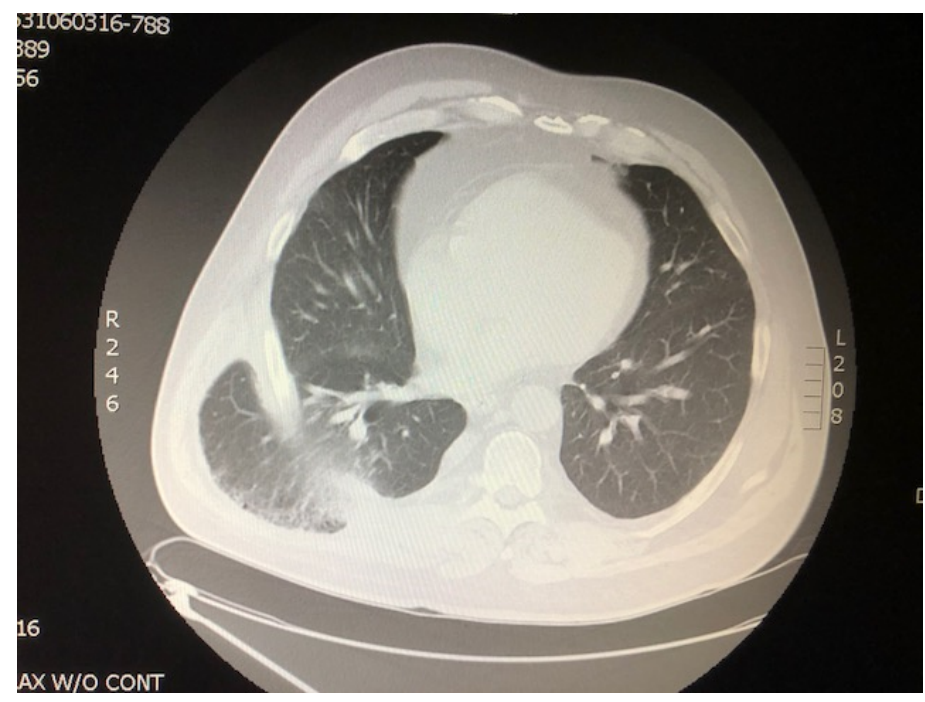

Figure 2B. CT of chest, non-contrast, lung windows, axial view at similar level as Figure 2A

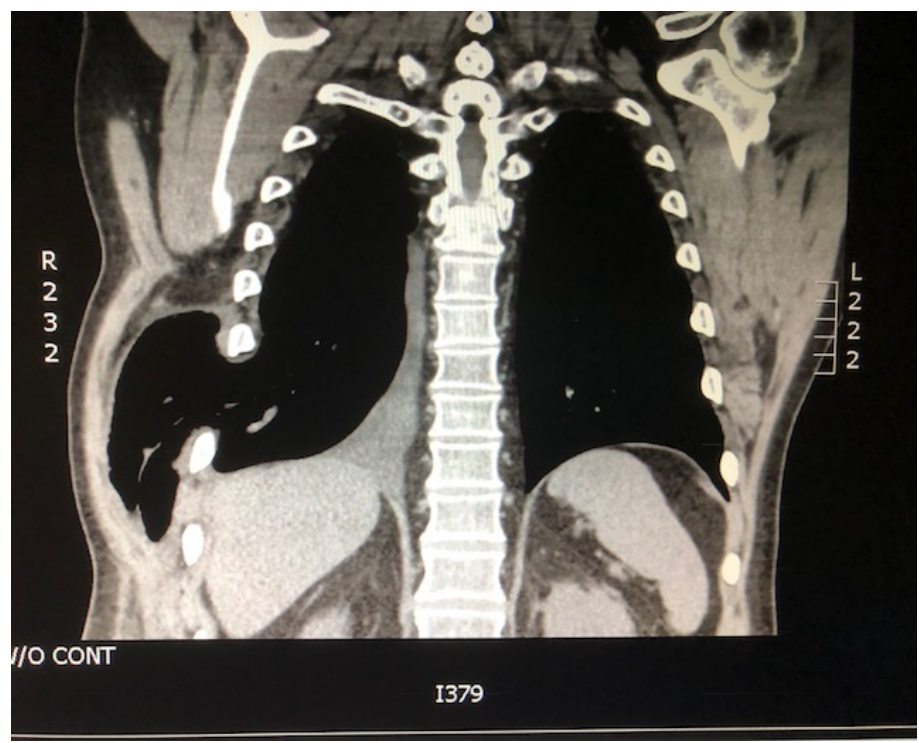

Figure 3. Chest $\mathrm{CT}$, coronal view 


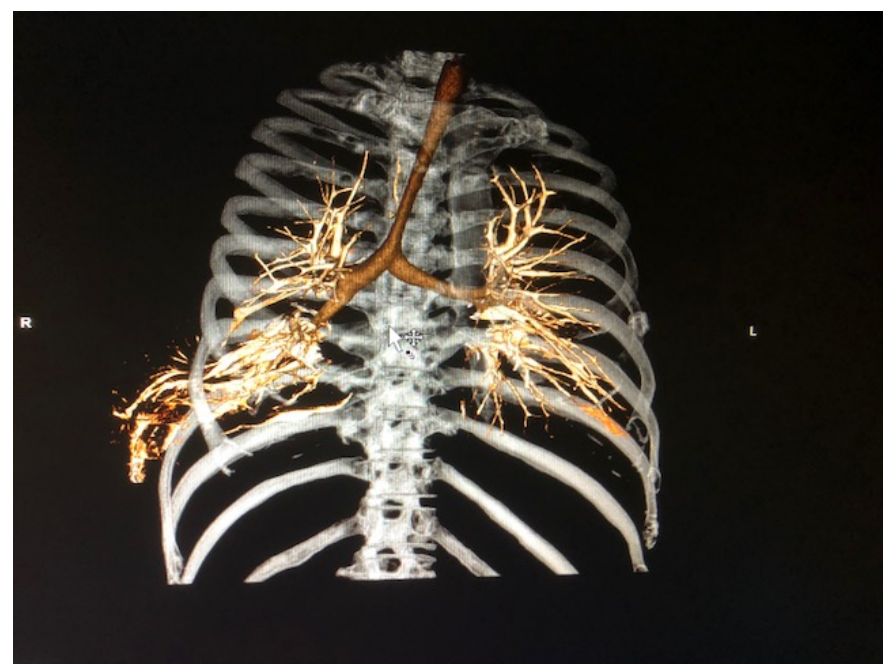

Figure 4. Virtual reality images showing bronchial and vascular anatomy with extrusion through chest wall defect

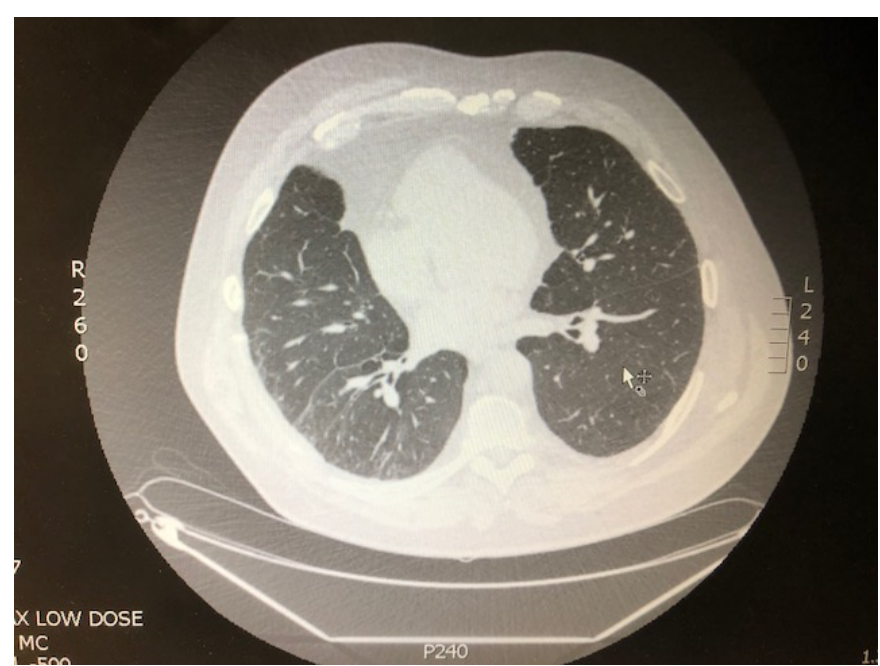

Figure 5. Chest CT, axial view, obtained after surgical repair of chest wall defect

intrathoracic pressures with defects in the thoracic wall and is usually diagnosed following trauma or chest surgery [1]. Spontaneous lung herniation is rare and has been reported following coughing, sneezing, playing a wind instrument, or heavy lifting that may have caused damage to a rib or cartilage [2-4]. These events have been most common in males, particularly those that are smokers or are obese, such as the patient in this case. Not all defects require surgical repair. Indications for surgery include increasing size, pain, and potential incarceration of lung tissue. This patient likely ruptured muscle fibers during heavy lifting, with cough-induced herniation of a large section of lung tissue through the defect.

\section{References}

1. Ishida A, Oki M, Saka H, Seki Y (2018) Postoperative intercostal lung hernia. Respirol Case Rep 6: e00323. [Crossref]

2. O'Shea M, Cleasby M (2012) Images in clinical medicine. Lung herniation after coughinduced rupture of intercostal muscle. N Engl J Med 366: 74. [Crossref]

3. Sulaiman A, Cottin V, De Souza Neto EP, Orsini A, Cordier JF, et al. (2006) Coughinduced intercostal lung herniation requiring surgery: Report of a case. Surg Today 36: 978-980. [Crossref]

4. Tack D, Wattiez A, Schtickzelle JC, Delcour C (2000) Spontaneous lung herniation after a single cough. Eur Radiol 10: 500-502. [Crossref]

Copyright: $\ 2018$ Carvalho AM. This is an open-access article distributed under the terms of the Creative Commons Attribution License, which permits unrestricted use, distribution, and reproduction in any medium, provided the original author and source are credited. 